(Aus der pathologischen Abteilung des Karolinischen Instituts [Vorstand: Prof. Dr. Sundberg] und der bakteriologischen Abteilung der medizinischen Staatsanstalt [Vorstand: Prcf. Dr. Peterson] zu Stockholm.

\title{
Zur Pathogenese der sogenannten Weilschen Krankheit: Ein Fall von Proteusinfektion beim Säugling.
}

\author{
Von \\ Folke Henschen und John Reenstierna, Stockholm.
}

(Eingegangen am 18. März 1916.)

Beim Studium der Literatur über die sog. Weilsche Krankheit bemerkt man bald, daß die unter diesem Namen bekannten Fälle wenig einheitlich sind. Auch wurde die Natur dieser „Krankheit" als eine Krankheit sui generis schon wenige Jahre nach der ersten Mitteilung Weils ${ }^{1}$ ) von einer Reihe von Forschern in Abrede gestellt. Die Weilsche Krankheit wurde im Laufe der Jahre bald mit der einen, bald der anderen, ätiologisch verschiedenartigen Form von Ikterus verwechselt oder identifiziert. Mit vollem Recht schlägt deshalb Gia ni 1905 vor, daß man nicht mehr von einer ,Weilschen Krankheit", sondern nur von einem „Weilschen Symptomenkomplex" sprechen sollte, und Ebstein läßt, ebenfalls 1905, die Bezeichnung ,Weilsche“ Krankheit vollständig fallen.

Auf die Lehre von der Symptomatologie der Weilschen Krankheit sei hier nicht eingegangen, insbesondere weil der vorliegende Fall hauptsächlich aus pathologisch-anatomischen und bakteriologischen Gesichtspunkten behandelt wird. Nur darauf soll die Aufmerksamkeit gelenkt werden, daß die von Klinikern mehrfach hervorgehobene Ähnlichkeit, ja selbst Identität der Krankheit mit dem aus Ägypten bekannten, sog. biliösen Typhoid auch in der pathologisch-anatomischen Literatur zum Vorschein kommt. So schreibt Ka uf ma n n (1911): ,Als biliöses Typhoid oder Weilsche Krankheit (Icterus infectiosus) wird eine fieberhafte Erkrankung bezeichnet ...".

1) Über eine eigentümliche, mit Milztumor, Ikterus und Nephritis einhergehende, akute Infektionskrankheit. Archiv f. klin. Med. 1886.

Zeitschrift für Kinderheilkunde. O. XIV. 
Daß die Weilsche Krankheit auch bei Kindern vorkommt, geht u. a. aus den Mitteilungen Baginskys und Brünings hervor.

Ätiologisch verhalten sich die unter dem Namen Weilsche Krankheit bekannten Fälle sehr verschieden. Der bakteriologische Befund, auf den wir unten zurückkehren, wechselt innerhalb weiter Grenzen. In einer Reihe von Fällen wurden Proteusarten gefunden. In zwei Fällen (Ba n ti und Gia ni) soll der Erreger ein dem Friedländerschen nahestehender Bacillus gewesen sein. In vielen Fällen hat es sich zweifelsohne um Typhus mit Ikterus gehandelt. Ka uf man n scheint eine primäre Typhusinfektion der Gallenwege anzunehmen. Zur Paratyphusgruppe wurde der Erreger der Weilschen Krankheit in einigen Fällen gerechnet. Bisweilen dürfte eine Mischinfektion vorhanden gewesen sein. Eine Sonderstellung nehmen Hecker und Otto (1911) ein: Der Erreger der Krankheit wäre noch unbekannt; wahrscheinlich handele es sich um ein durch Insekten verbreitetes, invisibles Virus.

Bei einer so wechselnden Ätiologie und Nosologie hat man auch keine einheitliche pathologische Anatomie zu erwarten. Eben auf Grund des verschiedenartigen Sektionsbefundes schien es schon $\mathrm{Nau}$ werk, A. Fraenkel und Mazzotti (1888/89), weniger gut begründet, die ,Weilsche Krankheit" als eine spezielle Infektionskrankheit aufzustellen.

Ätiologisch wenig einheitlich, klinisch unbestimmt und pathologischanatomisch wechselnd tritt däs Bild der Weilschen Krankheit in der Literatur hervor. Gerne stimmt man A. Posselt (1914) zu: „Der Name ,Weilsche Krankheit' hat nur einen recht bedingten, rein klinischsymptomatologischen und hier auch wieder vielfach anzukämpfenden Standplatz."

\section{Klinisches.}

Knabe, geb. 15. IX. 1913; Gewịcht bei der Geburt 3250 gm. Übrige Geschwister gesund. Muttermilch zwei Tage, dann künstliche Nahrung: Milch 2 T. + Wasser 6 T. + Maltose 3 Teel., auf 5 Flaschen verteilt. Anderthalb Woche nach der Geburt Gelbfärbung, die nach einer Woche schwand. Kam allmählich herunter: Erbrechen, schlecht verdaute schleimige Stühle, Schnupfen. - Am 17. XI. wieder allgemeine Gelbfärbung; Allgemeinzustand verschlechtert, schlechte EB. lust, wiederholtes Erbrechen.

Aufnahme 20. XI. ins Sachssche Kinderkrankenhaus (Chefarzt Dr. Ernberg).

Körpergewicht $3750 \mathrm{~g}$, Temp. 35,6. Algemeinzustand sehr schlecht; in tensive allgemeine Gelbfärbung der Haut und der Schleimhäute. Am Gesäß leicht blutende Intertrigo. Ödem der Fußrücken und der Lider; Conjunctivitis. Fettpolster schlaff und spärlich. Leichte Rigidität. Blutiges Erbrechen. Gelinder Schnupfen; doppelseitige eitrige Mittelohrentzündung. Herz ohne Besonderheiten. 
Lungen: beiderseits Rhonohi. Bauch etwas groß, die Milz palpabel (?), Leberdämpfung nicht vergrößert. Hals- und Leistenlymphknoten leicht vergrößert, Har n gelbgrün, enthält Gallenfarbstoff, Albumen und massenhaft Leukocyten; keine Zylinder, keine reduzierende Substanz. Zwei hellgraue, schlecht verdaute, übelriechende Stühle. - Paracentese; rechts reichliche Blutung nach derselben. 21. XI. Leichte Blutung aus der Nase. Temp. 36,7. Exitus um 8 Uhr vorm.

K. D. Morbus Weilii, Otitis media purulenta bilateralis. Bronchitis acuta. Nephritis acuta.

\section{Pathologisch-Anatomisches.}

Sektion 22. XI. um 9 Uhr vorm. (Henschen).:

Körpergewicht 3620 g. Kleiner abgemagerter Knabe. Hautfarbe stark gummiguttagelb. Am Gefäß Intertrigo und punktförmige subcutane kleine Blutungen. Sichtbare Schleimhäute stark gelb, ziemlich blaß. Die Th y m us klein, schlaff und ikterisch. Herzbeutel ohne Besonderheiten. Das Herz etwas groß, sein Gewicht $38 \mathrm{~g}$. Die Muskulatur ziemlich dick und fest. Die hintere und rechte. Aortenklappe größtenteills in eine einzige große, etwas dicke Klappe aufgegangen. An den Schluß. rändern der: Mitral - und Tricupspidalklappen kleine lebhaft rote Pünktchen. Das Endokard etwas undurchsichtig, gleichmäßig gelb. Das Foramen ovale ist für einen Bleistift durchgängig. Die Aorta stark ikterisch, ebenso die Pulmonalis. Der Ductus botalli läßt eine feine Sonde durch. Die linke Ple ura ohne Besonderheiten. Rechts ist die Lunge mittelst fester, zäher Adhäsionen mit der Brustwand verwachsen. Ähnliche Verwachsungen intralobär. Die linke Lunge ikterisch, hyperämisch. Die hinteren Teile der beiden großen Lappen der rechten Lunge fest und kompakt. Aus dem dunkelgelbroten Parenchym wird ziemlich spärliche, trübe, luftfreie Flüssigkeit ansgepreßt. Doppelseitige schleimig-eitrige Bronchitis. Katarrhalische Tracheitis und Pharyngitis; Ikterus der Schleimhäute. Bauchhöhle ohne Besonderheiten. Die Milz vergrößert und fest, ihr Gewicht 22 g. Die.Pulpa fest, dunkelgelbrot. Die. Follikel nicht sichtbar. Nebennieren stark ikterisch. Die Nieren vergrößert, schlaff aber eigentümlich gespannt. Das Parenchym schwillt erheblich. Die Rinde größtenteils ungewöhnlich breit, ihre Zeichung fast günzlich verwischt. Die Farbe trübe, graugelblich. Die Zeichnung der Pyramiden deutlich. Das Bindegewebe des Nierenbeckens stark ikterisch, die. Schleimhaut des Beckens blaß, mit einer reiçlichen eiterähnlichen Masse bedeckt. Die Ureteren leicht erweitert. Die Blase enthält stark trüben ikterischen Harn; ihre Schleimhaut blaß. Die Speiseröhre und der Magen ohne Besonderheiten. Im Darm spärlicher schleimiger brauner Inhalt. Perinoduläre Pseudomelanose im Kolon. Die Darmschleimhaut mäßig ikterisch, sonst ohne Besonderheiten. Die Leber nicht deutlich vergrößert, von gewöhnlicher Konsistenz; ihre Oberfläche eben; die Farbe dunkel olivengrün, fast ganz schwarz. Die Schnittfläche eben, feucht; ihre Zeichnung tritt durch eine dunklere schwarzgrüne Farbe der peripherischen Acinusteile deutlich hervor. Durch ihre hellere gelbliche Farbe werden die Gallengänge und das gröbere perivasculäre. Bindegewebe deutlich hervorgehoben. In der Gallenblase 1-2 ccm klare, vollständig farblose, etwas zähe Flüssigkeit, die ins Duodenum leicht herausgepręit wird. Die gröberen Gallengänge und die Papilla Vateri ohne Besonderheiten. Das Pankreas ikterisch, sonst ohne Besonderheiten. Die harte Hirnhaut stark ikterisch, die weichen Häute nur wenig gelb, dagegen ziemlich 
blutreich und ödematös. Die Hirnsubstanz ohne Besonderheiten. Keine ikterische Verfärbung der Zentralganglien. Im Mittelohr beiderseits trübe, schleimige Flüssigkeit, die links auch hämorrhagisch ist.

P. A. D. Hypertrophia cordis, Degeneratio parenchymatosa myocardii. Pleuritis et pneumonia dextraveterior, Bronehitis muco-purulenta. Hyperplasia lienis. Nephritis acuta. Icterus gravis hepatis, Hydrops vesicae felleae. Otitis media purulenta bilateralis. Icterus universalis gravis.

Stückchen der Leber sowie Blut aus der rechten und linken Vena femoralis wurden für bakteriologische Untersuchung steril genommen. Teile des Herzens, der Lungen und Nieren, der Milz, des Darms, der Leber und Gallengänge und des Pankreas wurden in 10proz. Formol und in Zenkers Flüssigkeit konserviert.

\section{Mikroskopisehe Untersuchung.}

Herz: Keine bemerkenswerten Veränderungen bei den gewöhnlichen Färbungen; auch die Sudan-Färbung fiel negativ aus.

R. Lunge: Stark konfluierende bronchopneumonische Herde von geringem Fibringehalt und stark wechselndem Zellgehalt; bald massenhaft polymorphkernige Leukocyten, bald nur spärliche Leukocyten, bald wieder fast nur große mononucleäre Zellen in den Alveolen; leichte Bindegewebswucherung, Hyperämie; starke eitrige Bronchitis.

Milz: Lymphknötchen klein, enthalten nur ausnahmsweise deutliche Keimzentren. Die Pulpa ziemlich hyperämisch, enthält gelapptkernige Leukocyten in mäßiger, wechselnder Menge, Myelocyten - fast nur neutrophile-- und verhältnismäßig zahlreiche Plasmazellen. Blutkörperchenhaltige Zellen kommen auch vor, ebenso ist eine Vermehrung der Pulpazellen festzustellen. Pigment konnte nicht sicher gesehen werden.

In den mesenterialen und portalen Lymphknoten ist die grobhistologische Zeichnung sehr verwischt; die meistens ödematösen Knoten zeigen eine bedeutende leukocytäre Infiltration und Desquamation des Sinusendothels.

Die Nieren sind vielleicht am stärksten verändert: Das ganze Parenchym zeigt eine sehr bedeutende allgemeine leukocytäre Infiltration. Auffallend ist jedoch, daß die Malpighischen Körperchen fast vollständig verschont worden sind. Dagegen enthalten die Lumina der Kanälchen überall Leukocyten, die oft in so großer Menge vorhanden sind, daß die Kanälchen stark ausgedehnt sind. Dabei zeigen die Leukocyten nicht selten Entartungserscheinungen verschiedener Art. Interstitiell kommen die Leukocyten teils ganz diffus, teils in dichteren Häufchen vor, ohne daß es irgendwo zu wirklicher Absceßbildung gelangt. Die Epithelien der gewundenen und geraden Kanälchen enthalten bisweilen Tröpfchen in nicht geringer Menge, die sich mit Sudan lebhaft rot färben; auch interstitiell findet sich dann und wann spärliche sudangefärbte Substanz in Tropfenform in kleinen rundlichen Zellen; doppelbrechende Substanz konnte nicht nachgewiesen werden. Im Lumen der Kanälchen kommen auch Gallenfarbstoffzylinder vor, obgleich in spärlicher Menge. In den oberflächlichsten Teilen der Rinde sieht man ab und zu kleine A nsammlungen dicht liegender Stäbchen von etwas plumper Form. Der Gram. Färbung gegenüber verhalten sie sich negativ. Bisweilen be- 
obachtet man eine besonders dichte Anhäufung von Leukocyten in ihrer Umgebung.

Der Darm bietet keine bemerkenswerten Veränderungen dar. Leider konnte nur der Dünndarm untersucht werden.

Die Leber enthält keine mit Sudan färbbare Substanz. Gallenpigment kommt ziemlich reichlich vor; die zentralen Teile der Lobuli sind dabei bevorzugt; das Pigment tritt teils als eine fast diffuse grüngelbe Verfärbung der Zellen auf, teils in Form von kleinen und großen amorphen Körnchen und Schollen. In den Gallen. capillaren findet man fast keine Schollen. Die Leberzellbalken sind vielleicht etwas schmal, die Zellen der zentralsten Teile der Läppchen bisweilen kleiner als gewöhnlich. Das interlobuläre Bindegewebe zeigt ab und zu leichte kleinzellige Infiltration. Die intralobulären Blutcapillaren sind meistens erweitert und enthalten neben roten Blutkörperchen und großen Mengen von Lymphocyten, Myeloblasten, Myelocyten und Leukocyten.

Die gröberen Gallengänge und die Gallenblase bieten keine bemerkenswerten Veränderungen dar; die interlobulären Gallengänge sind ebenfalls ohne Bemerkung.

Das Pankreas bietet nichts Bemerkenswertes dar.

Die pathologisch-anatomischen Veränderungen betreffen also fast sämtliche Organe des Körpers. Die Veränderungen der rechten Pleura, der Lungen, der Luftwege und Mittelohren bieten wohl an sich kaum etwas von besonderem Interesse dar und entsprechen, da sie wenigstens zum Teil längere Zeit bestanden, einer früheren Infektion. Zum Teil sind sie doch ohne Zweifel ganz frisch. Die übrigens recht geringe Herzhypertrophie kann mit den älteren Lungenveränderungen im Zusammenhang stehen, könnte aber auch von den kleinen Anomalien am Herzen selbst hervorgerufen sein. Die Milzveränderungen schließen sich wohl ziemlich genau an den gewöhnlichen Befund bei subakuten und akuten Infektionen des frühen Kindesalters an.

In den mesenterialen Lymphdrüsen finden sich ziemlich erhebliche subakute und akute Veränderungen, die ohne Zweifel stärker sind als bei den gewöhnlichen Gastroenteritiden dieses Alters. Auffallend ist nur, daß die untersuchten Darmteile keine auffallenden Veränderungen darbieten. Das Kolon wurde indessen nicht untersucht.

Die Leber bietet auch mikroskopisch kein anderes Bild dar als dasjenige eines ziemlich starken Ikterus. Dabei ist zu bemerken, daß Zeichen einer Gallenstauung innerhalb der Capillaren kaum vorhanden sind. Auch war der spärliche Darminhalt bei der Selction deutlich gallig gefärbt; klinisch wurden indessen einmal ,, hellgraue, schlecht verdünnte, übelriechende Stühle" bemerkt. Ein Stauungsikterus scheint deshalb kaum vorzuliegen ${ }^{1}$ ). In dieser Hinsicht sind die Untersuchungen von

1) Der bei der Sektion beobachtete geringe Hydrops der Gallenblase stellt einen bei kleinen Kindern so häufigen Befund dar, daß man demselben keine 
Ugolini und Mazzocchi von Interesse: Auch von ihnen wurde die Abwesenheit einer Gallenstauung innerhalb der Capillaren festgestellt, und zwar, in ihrem Fall, bei acholischem Darminhalt. Die Verfasser meinen, daß es sich in solchen Fällen um eine veränderte Funktion der Leberzellen handeln würde. Analoge Verhältnisse hinsichtlich der Gallencapillaren " sind nicht unbekannt (z. B. bei der kongenitalen Lebercirrhose).

Zeichen irgendeiner Hepatitis waren in unserem Falle nicht vorhanden, weder in der Form stärkerer parenchymatöser Veränderungen, noch in der Form einer interstitiellen Entzündung. Die oben erwähnte kleinzellige Infiltration des Bindegewebes dürfte nur eine für das Alter physiologische Erscheinung sein. Eine Leberschwellung wurde weder klinisch noch anatomisch gefunden. In einer Reihe von Fällen, die unter dem Bilde der Weilschen Krankheit verliefen, wurden tiefgreifende hepatitische Veränderungen festgestellt, die in den schwersten Formen an die subakute und akute gelbe Leberatrophie erinnerten (z. B. Guthrie, de Luna, Mrosik). Klinisch können die akuten Leberveränderungen in einer erheblichen Schwellung des Organs zum Vorschein kommen (Einhorn).

Nicht ohne Interesse sind die erheblichen Mengen von weißen Blutkörperchen innerhalb der Lebercapillaren. Zweifelsohne deuten sie auf eine bedeutende allgemeine Vermehrung der Blutleukocyten hin. Auf eine nähere Analyse der intravitalen Blutveränderungen muß man wohl verzichten, trotzdem eben die Lebercapillaren besonders günstig sind für das postmortale Studium derselben. Das reichliche Auftreten von unreifen Zellen steht wahrscheinlich mit dem Alter in Zusammenhang.

Am interessantesten und wichtigsten scheinen die Nierenveränderungen zu sein. Klinisch wurde Eiwei $\beta$ und massenhafte Leukocyten gefunden. Makroskopisch boten die Nieren das Bild einer sehr schweren akuten Alteration dar. Mikroskopisch am meisten auffallend ist die sehr starke allgemeine Infiltration mit gelapptkernigen Leukocyten und die reichliche Ausscheidung von Eiterkörperchen. Die eiterähnlichen Massen der Nierenbecken bestanden wohl zum größten Teil aus ausgeschiedenen Leukocyten. Kein Wunder, daß die Nierenepithelien stark gelitten haben! Von besonderem Gewicht ist natürlich der Nachweis von Bakterien am Schnitt. Alles spricht dafür, däß die gefundenen Mikroorganismen mit dem bei der Züchtung gewonnenen Proteus (s. unten) größere Bedeutung für den vorliegenden Fall beimessen dürfte. (Vgl. die äußerst gewöhnliche Erweiterung der Ureteren und Nierenbeckén bei Kindern.) 
identisch sind. Morphologisch-tinktoriell sind sie von demselben nicht zu trennen. Die ganz bestimmte Lage der Bakterienhäufchen im Parenchym, vor allem aber die wallförmige Anordnung der Leukocyten, sprechen mit Bestimmtheit für die intravitale Natur dieser Kolonien. In diesem Zusammenhang sei daran erinnert, daß Jaeger in mehreren Fällen vom Morbus Weilii aus dem Harn einen Proteus gewinnen konnte. Ảhnliche Befunde werden von Conradi und Vogt und von Machinsky erhoben. Brüning züchtete denselben Bacillus aus dem Harn eines viermonatigen Säuglings.

Mikroskopische Untersuchungen über das Verhalten der Nieren bei der sog. Weilschen Krankheit liegen in mehreren Fällen vor. Charakteristisch wäre nach A. Fraenkel (Eulenburgs Realenzyklopädie) trübe Schwellung, fettige Entartung, nekrobiotischer Untergang der Zellen und kleinzellige Infiltration des Bindegewebsgerüstes. In einem Fall von Münzer hatte die Nephritis einen hämorrhagischen Charakter angenommen. - Auf die Nierenveränderungen der verschiedenen Fälle näher einzugehen, scheint nicht zweckmäßig, da sie wohl mit der wechselnden Ätiologie in intimem Zusammenhang stehen. Jedenfalls scheinen die Nierenveränderungen des vorliegenden Falles ganz besonders bösartig gewesen zu sein.

Bakteriologisches (Reenstierna).

Aus der Leiche wurden (24 Stunden nach dem Tode) der Züchtung wegen unter sterilen Kautelen je eine Blutprobe aus der linken und rechten Vena femoralis und ein cm-Stückchen Leber entnommen. Hiervon wurde Aussaat in folgende Nährbödèn gemacht: gewöhnlichen Agar, Ascitesagar, gewöhnliche Bouillon und Ascitesbouilion. In sämtlichen Substraten wuchsen nach 24 Stunden (bei $37^{\circ}$ ) stäbchenförmige Bakterien, welche bei genauerer Untersuchung als Reinkulturen sich erwiesen. Man bekam auBerdem gleich den Eindruck, daß die von den drei verschiedenen Quellen herrührenden Bakterien identisch waren.

Bei der Beschreibung der Stämme sind folgende Abkürzungen benutzt:

$\mathbf{B}_{1}=$ Bakterie aus der Vena femoralis sin. gezüchtet;

$\mathbf{B}_{2}=$ Bakterie aus der Vena femoralis dextr. gezüchtet,

$B_{3}=$ Bakterie aus der Leber gezüchtet.

$B_{1}, B_{2}$ und $B_{3}$ sind Bakterien mit einem gewissen Polymorphismus: ganz kurze Stäbchen bis lange Fäden. Sie färben sich gut mit den gewöhnlichen Anilinfarben, werden nach Gram entfärbt, sind kapseltragend, und machen im hängenden Tropfen sehr leḅhafte Bewegungen. Sie wachsen sowohl aërob wie anaërob. Der Agarnährboden bekommt binnen 24 Stunden (bei $37^{\circ}$ ) einen saftigen deutlich grünfluorescierenden Belag. Die Kolonien in Agarplatten haben nichts besonders Charakteristisches. Einzelne Schwärmerkolonien wurden jedoch gesehen. In Gelatineplatten zeigen nach einigen Tagen mehrere Kolonien schalenförmige Vertiefungen (Peptonisierung). Der Gelatinestich gibt eine, im Anfang oben schalen- 
förmige Einschmelzung. Nährbouillon wird stark getrübt, und dann entsteht ein mächtiger Bodensatz. Bei Kartoffeln entsteht ein ziemlich saftiger, gräulicher bis bräunlicher Belag. Milch wird koaguliert. Das Gerinnsel löst sich später; die Reaktion bleibt neutral. Traubenzucker - nicht aber Milch- und Rohrzucker wird unter Gasbildung vergoren. In gewöhnlicher Bouillon mit 0,5\% Harnstoff ist schon am zweiten Tage starke Ammoniakentwicklung zu bemerken, in solchen ohne Harnstoff wird ein eingehängtes rotes Lackmuspapier erst nach drei Tagen schwach blau gefärbt.

Mit den drei Bakterienstämmen wurden Kaninchen immunisiert und das gewonnene Serum für Agglutination verwendet:

$B_{1}$ - Ser um agglutiniert $B_{1}-$ Stamm $1: 128000, B_{2} 1: 16000, B_{3} 1: 128000$;

$B_{2}$ - Serum: $B_{1} 1: 256000, B_{2} 1: 256000, B_{3} 1: 64000$;

$B_{3}$ - Serum: $B_{1} 1: 256000, B_{2} 1: 2000, B_{3} 1: 256000$.

Agglutinationsversuche mit diesen Sera gegen einen Typhus-, einen Paratyphus- und einen Proteus-mirabilis-Stamm fielen negativ aus. Ein Stamm Proteus vulgaris $\mathrm{Kral}$ wurde dagegen agglutiniert: von $\mathrm{B}_{1}$-Serum $1: 16000, \mathrm{~B}_{2}$-Serum $1: 32000, B_{3}$-Serum $1: 16000$.

Vom Patienten war kein Blut entnommen worden, und konnten deswegen Agglutinationsversuche damit nicht angestellt werden.

\section{Tierversuche ${ }^{1}$ ).}

Meerschweinchen. Einige Tage nach der Reinzüchtung der drei Stämme aus der Leiche wurden 6 Meerschweinchen (2 für jeden Stamm) von je $250-300 \mathrm{~g}$ Körpergewicht geimpft. Jede Serie erhielt intraperitoneal $1 / 50 \mathrm{bzw} .1 / 20$ Normalöse. Ergebnis negativ.

Später wurden 9 etwa $200 \mathrm{~g}$ schwere Meerschweinchen mit den drei Stämmen intraperitoneal geimpft, und zwar mit folgendem Ergebnis. $B_{1}^{1 / 20}$ Normalöse: Versuchstier am folgenden Morgen tot. Sektion: Bauchfell injiziert; sonst nichts Bemerkenswertes. $B_{2}$ und $B_{3} 1 / 20$ Normalöse: die Tiere sterben nicht. $1 / 5$ und $1 / 2$ Öse sämtlicher Stämme töten die Tiere innerhalb 24 Stunden. Sektionsbefund: Bauchfell lebhaft gerötet; übrige Organe ohne Besonderheiten.

Aus äußeren Umständen wurden die folgenden Versuche erst etwa $1 \frac{1}{2}$ Jahre später angestellt:

\section{Kaninchen (der Immunisierung wegen):}

22. IV. 1915. Kaninchen I, Gewicht $2120 \mathrm{~g}, 1 / 2$ Normalöse $B_{1}$ intravenös 26. IV. 1915. " $1630 \mathrm{~g}, 1$ Normalöse $B_{2}$ intravenös 29. IV. 1915, tot " $1430 \mathrm{~g}$

Sektion sofort: An einer etwa $5 \mathrm{~cm}$ langen Partie vom distalem Drittel des Ileums ist sowohl die Serosa wie auch die Mucosa lebhaft injiziert. Sonst makroskopisch alles normal. In Kulturen von den verschiedenen Organen wurde ein Stäbchen erhalten, das mit dem eingeimpften, auch bei Agglutination, übereinstimmte.

Mikroskopische Untersuchung der Organe (Henschen):

Die Lungen in großer Ausdehnung pneumonisch. Innerhalb der Infiltrate große abgerundete Nekrosen, die fast immer dichte Knäuel von etwas plu m-

1) Bei sämtlichen Versuchen sind Agarkulturen (24 $\mathrm{St}$. bei $37^{\circ}$ ) angewendet. 
pen Stäbchen enthalten. Offenbar entsprechen die Bakterienhäufchen nicht selten Gefäßen. Die Stäbchen sind gramnegativ. Starke eitrige Bronchitis. Auch in der Milz findet man dieselben dichten Hä ufchen von gramnegativen Bak * terien. Die Milz zeigt starke Hyperämie. Die Nieren enthalten äußerst geringe Mengen von sudanfärbbarer Substanz und sind im übrigen fast unverändert; nur an einer Stelle war in der Papille ein kleines blaues Streifchen makroskopisch sichtbar, das sich als einige reihenförmig angeordnete Häufchen von Bakterien herausstellte; es handelte sich auch hier um gramnegative Stäbchen. Der Darm ohne Besonderheiten. Die Leber stellt sich bei der Sudanfärbung als fast fettfrei dar. In den intraacinären Capillaren finden sich ab und zu große Mengen dicht. gehäufter, gramnegativer Stäbchen.

29. IV. Kaninchen IV, Gewicht $2400 \mathrm{~g}, 1 / 4$ Normalöse, $B_{1}$ intravenös.

$$
\text { 1. V. tot }, 2060 \mathrm{~g}
$$

Sektion sofort: Am Duodenum vom Pylorus nach unten in einer Ausdehnung von etwa $3 \mathrm{~cm}$ sieht man Blutungen, welche sowohl von der Serosa wie der Mucosa aus sichtbar sind. Im oberen Drittel der betreffenden Partie ist es eine zusammenhängende Blutung, im übrigen ist dieselbe punktförmig und etwas größer. Die Organe sonst makroskopisch normal. Kulturell wurden die eingeimpfte Bakterie von verschiedenen Organen wiedergewonnen.

Mikroskopische Untersuchung der Organe (Henschen):

Einige dichtere und dunklere Partien der L ungen wurden näher untersucht: die Veränderungen beschränlkten sich indessen auf Atelektase und Hyperämie; daneben leichte katarrhalische Veränderungen. Die Milz ohne Besonderheiten. Nieren: ziemlich erhebliche fettige Entartung der Kanälchen, insbesondere der Schleifen. Ablagerung von sudangefärbter Substanz im interstitiellen Bindegewebe der Papillen. Hier und da in der Marksubstanz kleine dichte Ansam m. lungen von ziemlich großen Stäbchen, welche sich der Gramfärbung gegenüber negativ verhalten. Ringsum ist ein fast vollständig nekrotisoher Hof mit zerfallenden Leukocyten; peripherwärts davon starke leukocytäre Infiltration. Das Duodenum bietet - mit Ausnahme einer ziemlich verbreiteten frischen Blutung - keine Veränderungen dar. In der Leber findet sich Fett in mäßiger Menge, besonders in dem peripheren Teil der Läppchen. Die Gallenblase ohne Besonderheiten.

Kaninohen III und IV in derselben Weise mit $B_{2}$ bzw. $B_{3}$ geimpft (Anfangsdosis $1 / 2$ Normalöse) und Kaninchen $V$ (Anfangsdosis $1 / 10$ Normalöse B ${ }^{1}$ ). Alle Tiere überlebten die Immunisierung, magerten jedoch stark ab.

Affe: Kleiner weiblicher Macacus rhesus. Am 24. IV. 1915 abends mit $B_{1}$ geimpft: $1 / 2$ Agarkultur intravenös und $1 / 2$ intraperitoneal. Bei der intravenösen Injektion, die in eine Vene an der Hinterseite des linken Oberschenkels gemacht wurde, zuckte das Tier, wobei ein Teil der Bakterienemulsion (in physiologischer NaCl-Lösung) zufällig subcutan kam. 27. IV.: Tier sitzt zusammengekauert, sieht nicht wohl aus. Ungefähr derselbe Befund am 28. und 29. IV. 30. : Befinden sehr schlecht. An der Hinterseite des linken Oberschenkels bis an das Gesäß ist eine mißfarbige ödematöse Partie zu erkennen. 2. V. morgens tot.

Sektion sofort: Das betreffende Gebiet am linken Oberschenkel feucht und braunschwarz mißfarbig. Das Gewebe bei Incision bedeutend ödematös. Sämt- 
liche innere Organe makroskopisch ohne Befund. Kulturell wurde die eingeimpfte Bakterie wiedererhalten.

Mikroskopische Untersuchung der Organe (Henschen):

Die Lungen zeigen keine bemerkenswerten akuten Veränderungen. In der Milz sind die Lymphknötchen mäßig groß und meistens mit deutlichem Keimzentrum versehen. Die Pulpa ist sehr blutreich, enthält nur vereinzelte Leukocyten. Die Nieren enthalten keine mit Sudan färbbare Substanz und bieten auch sonst keinerlei Veränderungen dar. Die Leber ist fettarm; deutliche Veründerungen sind nicht vorhanden. Die Hirnsubstanz und die weichen Häute ohne Bèsonderheiten. Die veränderten Hauptpartien zeigen mikroskopisch starke leukocytäre Infiltration des subcutanen Fettgewebes mit angrenzender Muskulatur; die Haut ist in großer Ausdehnung vollständig nekrotisch; auch die Muskelfibrillen zeigen zum großen Teil hochgradige Entartung bis vollkommene Neurose. Bakterienfärbung ergibt indessen keinen entscheidenden Befund.

Hühner: Ein Hahn und zwei Hennen. 15. VII. 1915. Fütterungsversuche. Eine Mischung von Hafer und Zwieback wurde mit Bakterienemulsion (12 Agarkulturen, 4 von jedem Stamm, in physiologischer Kochsalzlösung) getränkt. Die Tiere, welche einen halben Tag lang kein Futter bekommen hatten, fraßen begierig obige Mischung. - Keine Wirkung.

Die bakteriologisch-serologischen Untersuchungen scheinen zu beweisen, daß diedrei a us der Leiché gezüchteten Stämme identisch sind und obne Zweifel der Proteus fluorescens-Familie angehören.

Es wurde also der fluorescierende Proteus aus den beiden V. femorales und der Leber in Reinkultur gewonnen. Daß dieser bakterielle Befund einer intravitalen Infektion entspricht und nicht durch irgendeine postmortale Einwanderung von Proteus hervorgerufen ist, dafür reden vor allem die mikroskopischen Nierenveränderungen : dichte Häufchen von proteusähnlichen Bakterien mit deutlicher vitaler, leukocytärer Reaktion des Nierengewebes.

Schon in der Einleitung wurde kurz angedentet, daß der Proteus fluorescens ein gar nicht seltener Befund bei der sog. Weilschen Krankheit ist. Von Jaeger, der wohl als erster diese Proteusinfektion nachgewiesen hat (1891), wurden eine Reihe von Fällen untersucht. In vier Fällen mit ausgesprochenem Weilschen Symptomenkomplex gelang es ihm, den Proteus aus dem Harn des Patienten zu züchten, und in drei Sektionsfällen gewann er den Proteus aus den Organen, und zwar in Reinkultur.

Silvestri und Daddi züchteten in einem Falle von infektiösem Ikterus einen Proteus capsulatus, und Adler wies in einem Fall von Weilscher Krankheit einen Proteus im Milzpunktat nach. Der oben erwähnte Fall von Brüning interessiert uns in diesem Zusammenhang ganz besonders, weil es sich um einen Säugling handelte. Auch die Fälle von 
Conradi und Vogt und von Machinski wurden schon oben erwähnt. Posselt hat einen Fall von Proteusinfektion der Gallenblase mit fieberhaftem Ikterus beobachtet.

Daß der Proteus in vielen Fällen von Weilscher Krankheit eine ganz bestimmte Rolle spielt, ist wohl kaum mehr zu leugnen. Andererseits scheint es ziemlich sicher, daß der Proteus gar nicht der einzige Erreger dieser Krankheit ist. In diesem Sinne hat die von Beitzke (in Brüning und Schwalbes Pathologie des Kindesalters) ausgesprochene Reservation ihre volle Bedeutung.

Die Frage nach der pathogenetischen Bedeutung der Proteusinfektion für die Weilsche Krankheit ist damit nicht erledigt. Eben im vorliegenden Falle scheint es, als würde die Proteusinfektion nicht das Ursprüngliche sein. Vielmehr wurden die anamnestischen Angaben einer früheren Infektion durch die Sektion gestützt. Das Kind war schon sehr heruntergekommen und der Boden sozusagen vorbereitet, ehe die Proteusinfektion zugestoßen ist. Während in unserem Falle der Erreger der früheren Infektion nicht entdeckt wurde, konnte in anderen Fällen festgestellt werden, daß es sich um eine Diphtherie-Proteus- oder InfluenzaProteusinfektion handelte. Vielleicht liegen ähnliche Verhältnisse vor in den von Whitaker beschriebenen Fällen mit Weilschen Symptomen während einer Influenzaepidemie.

Hinsichtlich des Auftretens von Ikterus gestalten sich verschiedene Proteusinfektionen sehr verschiedenartig. Es ist ja eine ganze Reihe von Fällen bekannt, wo die Proteusinfektion gar keinen Weilschen Symptomenkomplex hervorgerufen hat. Beispielsweise seien die Fälle von Baginsky (bei einem Kinde), von Bono me und Foà, von Rottka y erwähnt. Auch auf die Arbeiten von Haim und von Hübener könnte man verweisen. Vielleicht besitzen gewisse Proteusarten oder -stämme besonders stark hervortretende, ilkterogene" Eigenschaften (vgl. Bantis Bacillus icterogenes, der dem Friedländerschen nahestehen soll, von Jaeger aber als ein Proteus aufgefaßt wird). Bei den obenerwähnten Tierversuchen konnte indessen keine ,ikterogene" Beschaffenheit des vorliegenden Proteusstammes nachgewiesen werden.

\section{Literaturverzeichnis.}

In erster Linie sei auf folgende Arbeit verwiesen, die ein fast vollständiges Literaturverzeichnis enthält:

Posselt, A., Beziehungen zwischen Leber, Gallenwegen und Infektionskrankheiten. Anhang: Weilsche Krankheit. Lubarsch-Ostertag, Ergebnisse, XVII. Jahrg., II. Abteilung, S. 290. 
196 Zur Pathogenese der Weilschen Krankheit: Ein Fall von Proteusinfektion usw.

Daneben seien folgende Arbeiten hier aufgenommen, die bei Posselt nicht angeführt sind:

Baginsky, Archiv f. Kinderheilk. 12 (Proteusinfektion).

Beitzke, Die Infektionskrankheiten im Kindesalter. Brüning und Schwalbe,

Handb. d. Pathol. des Kindesalters, Wiesbaden 1912. 1, 225.

Bonome und Foà, zit. Großmann, Beiträge z. klin. Chir. 30 (Proteusinfektion). Dalche, Tcterus infectiosus. Soc. méd. des hôp., 1896, 29. Mai. Ref. Zentralbl.

f. Pathol. 59, 648.

Flesch, Jahrb. f. Kinderheilk. 60, H. 5. 1904.

Haim, Wiener klin. Wochenschr. 1903, S. 585 (Proteus).

Landouzy, Gaz. des hôp. 1883, S. 102, 115.

Weiß, Wiener med. Presse 1897, S. 889, 922 (Weilsche Krankheit). 